\title{
A Compensated Solenoid Giving a Uniform Magnetic Field Over a Large Volume
}

\author{
Chester Snow* and Raymond L. Driscoll
}

(October 7, 1964)

\begin{abstract}
The magnetic field any where inside a long solenoid with two symmetrically placed auxiliary windings is expressed in terms of zonal harmonics. Formulas are given that enable one to compute the size and position of the auxiliary windings that make the field most uniform at points in the neighborhood of the center of the solenoid.
\end{abstract}

Inquiries from workers in the field of nuclear resonance have indicated their need of a region of uniform magnetic field of considerable volume. The field at any point in a solenoid can be calculated with an accuracy consistent with one's knowledge of its geometry and current but the uniformity of field inside ideal coils of reasonable length is not sufficient over the volume required to develop a satisfactory nuclear resonance signal at the $10 \mathrm{G}$ level.

The following analysis shows how the variable part of the field near the center of a solenoid can be nearly compensated by two symmetrically placed auxiliary windings. This method of compensation has been suggested and used by others ${ }^{1}$ but a detailed analysis does not appear to have been published.

The solenoid is treated as a current sheet made up of $N$ turns of tape winding per unit length. The tape carries the current $I$, and the space between the turns of tape is assumed to be zero. The two equal and symmetrically placed auxiliary coils as shown in figure 1 with traces at $T_{1}, \bar{T}_{1}^{\prime}$, and $T_{1}^{\prime}, \bar{T}_{1}^{\prime}$ are treated as current filaments carrying the current $I_{1}$ each, where $I_{1}=N_{1} I$ and $N_{1}$ is a disposable constant. The winding channels of the two auxiliary coils must each have linear dimensions of its axial section, say $b$ and $d$, such that $b^{2} / a_{1}^{2}$ and $d^{2} / a_{1}^{2}$ are so small compared with unity as to be negligible to the precision desired in the magnetic field. The radius $a_{1}$ of each auxiliary coil and its axial position are to be determined so as to make the magnetic field most uniform near the origin. The equivalent filament that carries the current $I_{1}$ will have a trace that may be taken as the geometric center $T_{1}$ of the axial section of the winding channel.

The points $T_{1}$ and $\bar{T}_{1}$ are traces of a current filament of radius $a_{1}=r_{1} \sin \theta_{1}$ in the plane $x_{1}=r_{1} \cos \theta_{1}$. With spherical coordinates $r, \theta, \phi$ and cylindrical coordinates $x, \rho, \phi$ the only component of the vector potential is $A(r, \theta)=A_{\phi}(r, \theta)$ and this is independent of the longitude $\phi$. The spherical components of the magnetic field are

$$
\begin{aligned}
& H_{r}(r, \theta)=\frac{1}{r \sin \theta} \mathscr{D}_{\theta}[A(r, \theta) \sin \theta] \\
& H_{\theta}(r, \theta)=\frac{-1}{r} \mathscr{D}_{r}[r A(r, \theta)] .
\end{aligned}
$$

From these the cylindrical components are found by

$$
\begin{aligned}
& H_{x}=H_{r} \cos \theta-H_{\theta} \sin \theta \\
& H_{p}=H_{r} \sin \theta+H_{\theta} \cos \theta .
\end{aligned}
$$

*Retired, present address: 2520 Harrison Blvd., Ogden, Utah.
1 T. A. Heddle, Brit. J. Appl. Phys. 3, 95 (1952); B. Lewis, Brit. J. Appi. Phys. 1, 238 (1950); M. W. Garrett, J. Appl. Phys. 22 1091 (1951), M. F. Gardner, J. A. Jungerman. P. G. Lichtenstein. and C. G. Patten, Rev. Sci. Instr. 31, 929 (1960). 
The vector potential at $P(r, \theta)$ due to a cgs unit circular current with trace at $T_{1}\left(r_{1}, \theta_{1}\right)$ is given by

$$
\begin{aligned}
A(r, \theta) & =2 \pi \sin \theta \sin ^{2} \theta_{1} \sum_{n=1}^{\infty}\left(\frac{r}{r_{1}}\right)^{n} \frac{P_{n}^{\prime}(\cos \theta) P_{n}^{\prime}\left(\cos \theta_{1}\right)}{n(n+1)} \text { if } 0 \leq r \leq r_{1} \\
& =2 \pi \sin \theta \sin ^{2} \theta_{1} \sum_{n=1}^{\infty}\left(\frac{r_{1}}{r}\right)^{n+1} \frac{P_{n}^{\prime}(\cos \theta) P_{n}^{\prime}\left(\cos \theta_{1}\right)}{n(n+1)} \text { if } r \geq r_{1}
\end{aligned}
$$

where $P_{n}(u)$ is Legendre's polynomial and $P_{n}^{\prime}(u)$ denotes $\frac{d}{d u} P_{n}(u)$.

The effect of the two symmetrically placed equal filaments each with current $I_{1}$ is found from the first of these expansions, by superposition and noticing that in reckoning the effect of the filament on the left, the term $P_{n}^{\prime}\left(\cos \theta_{1}\right)$ must be replaced by $P_{n}^{\prime}\left(\cos \left(\pi-\theta_{1}\right)\right)=P_{n}^{\prime}\left(-\cos \theta_{1}\right)$ $=(-1)^{n+1} P_{n}^{\prime}\left(\cos \theta_{1}\right)$.

The vector potential at $P(r, \theta)$ of both current filaments is therefore

$$
A^{(1)}(r, \theta)=4 \pi I_{1} \sin \theta \sin ^{2} \theta_{1} \sum_{n=1}^{\infty}\left(\frac{r}{r_{1}}\right)^{2 n-1} \frac{P_{2 n-1}^{\prime}(\cos \theta) P_{2 n-1}^{\prime}\left(\cos \theta_{1}\right)}{2 n(2 n-1)} \text { when } 0 \leq r \leq r_{1}
$$

which applies therefore at points inside the solenoid near the origin.

By use of $\frac{d}{d u}\left[\left(1-u^{2}\right) P_{\nu}^{\prime}(u)\right]=-\nu(\nu+1) P_{\nu}(u)$ it is found that the field components are

$$
\begin{aligned}
& H_{r}^{(1)}=4 \pi I_{1} \frac{\sin ^{2} \theta_{1}}{r} \sum_{n=1}^{\infty}\left(\frac{r}{r_{1}}\right)^{2 n-1} P_{2 n-1}(\cos \theta) P_{2 n-1}^{\prime}\left(\cos \theta_{1}\right) \\
& H_{\theta}^{(1)}=-4 \pi I_{1} \sin ^{2} \theta_{1} \frac{\sin \theta}{r} \sum_{n=1}^{\infty}\left(\frac{r}{r_{1}}\right)^{2 n-1} \frac{P_{2 n-1}^{\prime}(\cos \theta) P_{2 n-1}^{\prime}\left(\cos \theta_{1}\right)}{2 n-1} .
\end{aligned}
$$

To obtain from these the expressions for the cylindrical components $H_{x}$ and $H_{\rho}$ it is necessary to make use of the two relations

and

$$
\left(1-u^{2}\right) P_{\nu}^{\prime}(u)+\nu u P_{\nu}(u)=\nu P_{\nu-1}(u)
$$

We find

$$
u P_{\nu}^{\prime}(u)-\nu P_{\nu}(u)=P_{\nu-1}^{\prime}(u)
$$

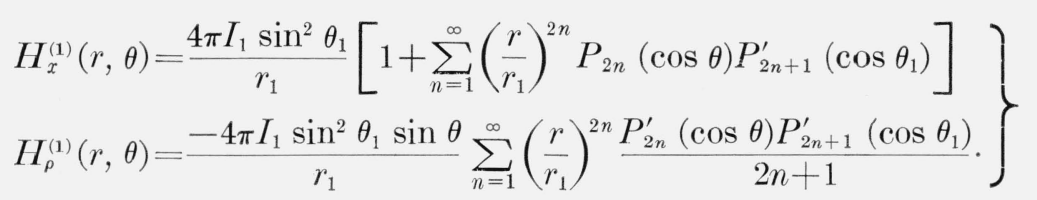

The vector potential of the current $I$ (cgs units) in the current sheet with $N$ turns.per cm is found by integration to be

$$
A^{(s)}(r, \theta)=4 \pi N \operatorname{Ir} \sin \theta\left[\frac{1}{2} \cos \alpha-\sin ^{2} \alpha \sum_{n=1}^{\infty}\left(\frac{r}{c}\right)^{2 n} \frac{P_{2 n+1}^{\prime}(\cos \theta) P_{2 n}^{\prime}(\cos \alpha)}{2 n(2 n+1)(2 n+2)}\right]
$$

at a point $P(r, \theta)$ inside the current sheet.

From this we find

and

$$
\left.\begin{array}{c}
H_{x}^{(s)}(r, \theta)=4 \pi N I\left[\cos \alpha-\sin ^{2} \alpha \sum_{n=1}^{\infty}\left(\frac{r}{c}\right)^{2 n} \frac{P_{2 n}(\cos \theta) P_{2 n}^{\prime}(\cos \alpha)}{2 n}\right] \\
H_{\rho}^{(s)}(r, \theta)=4 \pi N I \sin ^{2} \alpha \sin \theta \sum_{n=1}^{\infty}\left(\frac{r}{c}\right)^{2 n} \frac{P_{2 n}^{\prime}(\cos \theta) P_{2 n}^{\prime}(\cos \alpha)}{2 n(2 n+1)}
\end{array}\right\}
$$


For the case where the filament currents and solenoidal current circulate in the same sense around the $x$ axis we place $N_{1} I=I_{1}$ in eq (1) and add eq (2).

The resultant magnetic field inside the solenoid has the components

$$
\begin{gathered}
H_{x}(r, \theta)=4 \pi N I\left\{\cos \alpha+C_{0} \sin ^{2} \alpha-\sin ^{2} \alpha \sum_{n=1}^{\infty}\left(\frac{r}{c}\right)^{2 n} \frac{P_{2 n}(\cos \theta) P_{2 n}^{\prime}(\cos \alpha)}{2 n}\left(1-\frac{C_{0}}{C_{2 n}}\right)\right\} \\
H \rho(r, \theta)=4 \pi N I \sin ^{2} \alpha \sin \theta \sum_{n=1}^{\infty}\left(\frac{r}{c}\right)^{2 n} \frac{P_{2 n}^{\prime}(\cos \theta) P_{2 n}^{\prime}(\cos \alpha)}{2 n(2 n+1)}\left(1-\frac{C_{0}}{C_{2 n}}\right)
\end{gathered}
$$

where

$$
\begin{aligned}
C_{0} & \equiv \frac{N_{1} \sin ^{2} \theta_{1}}{N r_{1} \sin ^{2} \alpha} \\
C_{2 n} & \equiv\left(\frac{r_{1}}{c}\right)^{2 n} \frac{P_{2 n}^{\prime}(\cos \alpha)}{2 n P_{2 n+1}^{\prime}\left(\cos \theta_{1}\right)} \text { for } n=1,2,3, \ldots
\end{aligned}
$$

Also we may place here for reference

$$
\left.\begin{array}{l}
P_{2}^{\prime}(u)=3 u \\
P_{4}^{\prime}(u)=\frac{5 u}{2}\left(7 u^{2}-3\right) \\
P_{3}^{\prime}\left(u_{1}\right)=\frac{3}{2}\left(5 u_{1}^{2}-1\right) \\
P_{5}^{\prime}\left(u_{1}\right)=\frac{15}{8}\left(21 u_{1}^{4}-14 u_{1}^{2}+1\right)
\end{array}\right\} .
$$

For brevity, also let

$$
\lambda \equiv \frac{r_{1}}{c}, u=\cos \alpha \text {, and } u_{1}=\cos \theta_{1}
$$

where all three are positive (since $\theta_{1}$ refers to the filament on the right in fig. 1).

The variable part of $H_{x}$ is the series in (3a). If the first two terms corresponding to $n=1$ and $n=2$ are made to vanish, then the first two terms in the series (3b) for $H_{\rho}$ will also vanish. Therefore the approximately uniform resultant field near the origin will be in the axial direction.

The two equations $C_{2}=C_{0}$ and $C_{4}=C_{0}$ are

$$
\frac{\lambda^{3} P_{2}^{\prime}(u)}{2 P_{3}^{\prime}\left(u_{1}\right)}=\frac{N_{1}\left(1-u_{1}^{2}\right)}{c N\left(1-u^{2}\right)}=\lambda C_{0}>0,
$$

and

$$
\frac{\lambda^{5} P_{4}^{\prime}(u)}{4 P_{5}^{\prime}\left(u_{1}\right)}=\frac{N_{1}\left(1-u_{1}^{2}\right)}{c N\left(1-u^{2}\right)}
$$

Figure 1. Axial section of current sheet.

$c=$ the semi-diagonal of the section.

$\alpha=$ the semi-aperture of its end-circle viewed from $\mathrm{O}$

$N=$ number of turns per $\mathrm{cm}$ (tape-winding).

$I=$ the current circulating.

The points $\left(T_{1}, \bar{T}_{1}\right.$ (and) $T_{1}^{\prime}, \bar{T}_{1}^{\prime}$ ) are traces of the compensating filaments.

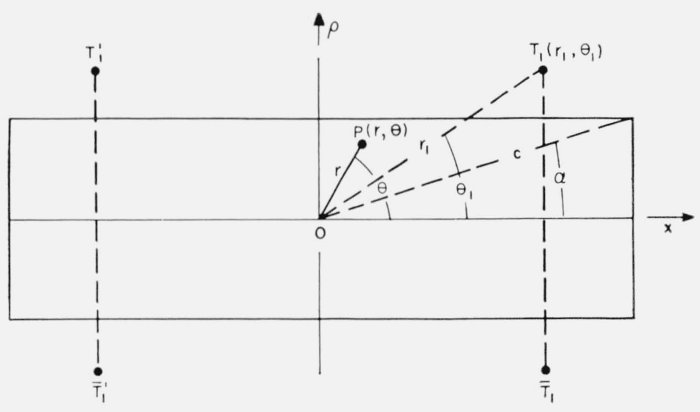


Dividing the members of eq (7) by the corresponding members of eq (8) gives

or, in view of eq (5),

$$
\lambda^{2}=\frac{2 P_{2}^{\prime}(u) P_{5}^{\prime}\left(u_{1}\right)}{P_{4}^{\prime}(u) P_{3}^{\prime}\left(u_{1}\right)}
$$

$$
\frac{r_{1}}{c}=\lambda=3 \sqrt{\frac{\left(u_{1}^{2}-1 / 3\right)^{2}-4 / 63}{5\left(u^{2}-3 / 7\right)\left(u_{1}^{2}-1 / 5\right)}}
$$

which must be real. We consider only the case of a relatively long solenoid in which $u=\cos \alpha>\sqrt{\frac{3}{7}}=0.6547$ or $0<\alpha<49^{\circ} 6^{\prime}$. In order that $\lambda$ be real it is necessary that the value of $u_{1}\left(=\cos \theta_{1}\right)$ to be found shall be such that

$$
\frac{\left(u_{1}^{2}-1 / 3\right)^{2}-4 / 63}{\left(u_{1}^{2}-1 / 5\right)}>0 .
$$

The zeros of the numerator of this fraction are

and

$$
\frac{1}{3}\left(1+\frac{2}{\sqrt{7}}\right)=0.5853
$$

$$
\frac{1}{3}\left(1-\frac{2}{\sqrt{7}}\right)=0.08136 \text {. }
$$

Since in eq $(9), u_{1}$ occurs in even powers only let

$$
Z=u_{1}^{2}
$$

Equation (9) may be written

$$
\frac{r_{1}}{c}=\lambda=3 \sqrt{\frac{(z-0.5853)(z-0.08136)}{5\left(u^{2}-\frac{3}{7}\right)(z-0.20)}} .
$$

If the value of $z$ to be found makes $\lambda$ real, this will correspond to the case that was adopted above in placing $I_{1}=+N_{1} I$ so the current filaments circulate around the $x$ axis in the same sense as the current $I$ in the solenoid. If values of $z$ are found that make $\lambda$ imaginary, a solution of the problem may still be found corresponding to $I_{1}=-N_{1} I$, which is equivalent to reversing the sign of $C_{0}$ so that $\lambda$ will then be real. If $\lambda$ as given by eq (11) is inserted in eq (7), and the relations of eq (5) employed, then

$$
\begin{aligned}
(1-z) P_{3}^{\prime}\left(u_{1}\right) & =\frac{15}{2}(1-z)(z-0.20)=\frac{c N\left(1-u^{2}\right) P_{2}^{\prime}(u) \lambda^{3}}{2 N_{1}} \\
& =\frac{3^{4} c N\left(1-u^{2}\right) u}{2 N_{1}}\left[\frac{(z-0.5853)(z-0.08136)}{5\left(u^{2}-\frac{3}{7}\right)(z-0.20)}\right]^{3 / 2} .
\end{aligned}
$$

Squaring eq (12) gives the following equation to determine $z$ (which also applies to the case in which $\lambda$ is imaginary):

$$
(1-z)^{2}(z-0.20)^{5}=B[(z-0.5853)(z-0.08136)]^{3}
$$

where

$$
B=3\left(\frac{3}{5}\right)^{5}\left(\frac{c N}{N_{1}}\right)^{2} u^{2}\left(1-u^{2}\right)^{2} .
$$

Hence $B$ is a positive constant if $u^{2}>\frac{3}{7}$ (or $\alpha<49^{\circ} 6^{\prime}$ ). The numerical solution of eq (13) may be found without difficulty when $B$ is given, that is, when the constants $c, N, N_{1}$, and $u=\cos \alpha$ are given. 
If we let

$$
F(z) \equiv \frac{[(z-0.5853)(z-0.08136)]^{3 / 5}}{(1-z)^{2 / 5}}
$$

and

$$
M=B^{-1 / 5}=\frac{5\left(u^{2}-\frac{3}{7}\right)^{3 / 5}}{3^{6 / 5}\left[\frac{C N u\left(1-u^{2}\right)}{N_{1}}\right]^{2 / 5}}
$$

equation (13) is equivalent to

$$
M(z-0.20)=F(z) \text { where } z=\cos ^{2} \theta_{1} .
$$

The roots of this equation between zero and one are the abscissas of the points of intersection of the curve $y=F(z)$ and the straight line $y=M(z-0.20)$. The curve $F(z)$ could be plotted once for all, since the constants $\alpha$ and $c$ of the solenoid enter only in the positive slope $M$ of the straight line. The general nature of the curve $y=F(z)$ is indicated in figure 2 . A fairly accurate plot of $F(z)$ and $M(z-0.20)$ versus $z$ is helpful in getting a starting point for an accurate evaluation of the roots by successive approximation. If $Z_{1}$ is a root, the corresponding value of $r_{1}$ is, by eq (12),

$$
\lambda^{3}=\left(\frac{r_{1}}{c}\right)^{3}=\left[\frac{5 N_{1}}{c N u\left(1-u^{2}\right)}\right]\left(1-Z_{1}\right)\left(Z_{1}-0.20\right)
$$

which must be positive. There will be two roots of eq (16): $Z_{1}$ in the interval $0.5853<Z_{1}<1$, $Z_{2}$ in the interval $0.08136<Z_{2}<0.20$. The latter is inadmissible when $I_{1}=+N_{1} I$.

FIGURE 2. The curves $\mathrm{y}=\mathrm{F}(\mathrm{z})$ and $\mathrm{y}=\mathrm{M}(\mathrm{z}-1 / 5)$ plotted as a function of $\mathrm{z}$.

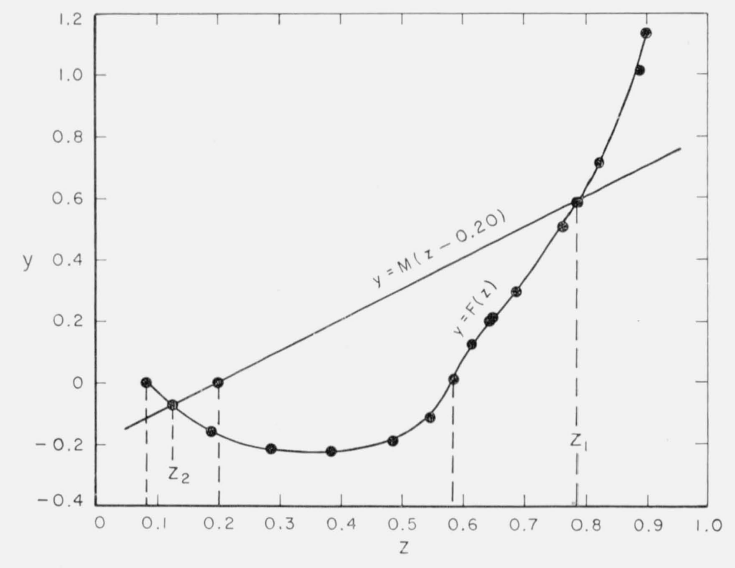

The root $Z_{1}$ gives the only value for $\cos ^{2} \theta_{1}$ permitted when the currents in the filament circulate around the $x$-axis in the same sense as the solenoidal current $I$. If in the opposite sense, $Z_{2}$ would be indicated. When the solenoid and the value of the constant $N_{1}$ are arbitrarily chosen, this solution gives the best radius and position of the two current filaments in order to produce a uniform field in a sphere with center at 0 . When the first two terms are thus annulled in the series of eqs (3a) and (3b), the next term $(n=3)$ will indicate the principal remaining: nonuniformity of field near the center by comparison with the constant term $\left(\cos \alpha+C_{0} \sin ^{2} \alpha\right)$ in eq (3a).

The foregoing analysis has been applied to the compensation of a solenoid designated as NBS 58080. The constants of this solenoid at $25^{\circ} \mathrm{C}$ are $N=9.99915$ turns per $\mathrm{cm}, c=51.9138_{7}$ $\mathrm{cm}$, and $\cos \alpha=0.963064$. For several assumed values of $N_{1}$ the constants of the required auxiliary windings have been found and listed in table 1 . For each value of $N_{1}$, the relative contribution of the compensating windings to the field at the center of the solenoid is shown in the column headed $C_{0} \sin ^{2} \alpha / \cos \alpha$. With the terms $n=1$ and 2 removed from eqs (3a) and (3b), the value of $r$, at which the term $n=3$ reaches one part per million (ppm) relative to the constant term $\left(\cos \alpha+C_{0} \sin ^{2} \alpha\right)$ in (3a) will be found in the table under "compensated radius." 
TABLE 1. Constants of auxiliary windings for solenoid NBS 58080

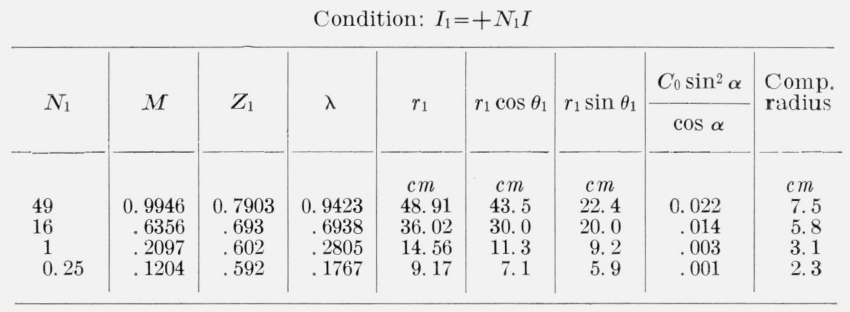

TABle 2. Constants of auxiliary windings for solenoid NBS 58080

\begin{tabular}{|c|c|c|c|c|c|c|c|c|}
\hline \multirow{2}{*}{$N_{1}$} & \multirow{2}{*}{$M$} & \multirow{2}{*}{$Z_{2}$} & \multirow{2}{*}{$\lambda$} & \multirow{2}{*}{$r_{1}$} & \multirow{2}{*}{$r_{1} \cos \theta_{1}$} & \multirow{2}{*}{$r_{1} \sin \theta_{1}$} & $C_{0} \sin ^{2} \alpha$ & \multirow{2}{*}{$\begin{array}{l}\text { Comp. } \\
\text { radius }\end{array}$} \\
\hline & & & & & & & $\cos \alpha$ & \\
\hline $\begin{array}{l}-49 \\
-16 \\
-1 \\
-0.25\end{array}$ & $\begin{array}{r}0.9946 \\
.6356 \\
.2097 \\
.1204\end{array}$ & $\begin{array}{r}0.1139 \\
.1006 \\
.0852 \\
.0829\end{array}$ & $\begin{array}{r}0.8017 \\
.5823 \\
.2438 \\
.1547\end{array}$ & $\begin{array}{c}c m \\
41.6 \\
30.2 \\
12.7 \\
8.03\end{array}$ & $\begin{array}{c}c m \\
14.05 \\
9.59 \\
3.69 \\
2.31\end{array}$ & $\begin{array}{l}c m \\
39.2 \\
28.7 \\
12.1 \\
7.7\end{array}$ & $\begin{array}{c}-0.108 \\
-.059 \\
-.0075 \\
-.0029\end{array}$ & $\begin{array}{l}c m \\
5.4 \\
4.6 \\
2.7 \\
2.0\end{array}$ \\
\hline
\end{tabular}

Corresponding values for the condition $I_{1}=-N_{1} I$ have been found and are listed in table 2 . There appears to be nothing in favor of the latter case, unless the relatively close spacing of the auxiliary windings is especially appropriate to an experiment. To give an idea of the expected improvement over the uncompensated solenoid, we calculate the term $n=1$ in the series of $H_{x}$ (eq (2)) relative to the constant term $\cos \alpha$, and find that it reaches $1 \mathrm{ppm}$ at $r=0.16 \mathrm{~cm}$.

We assume in what follows that the solenoid construction is accurate to $1 \mathrm{ppm}$. When deciding upon a design, one has to consider the accuracy required in the construction of the auxiliary coils in order to realize a given accuracy in the combination. If an accuracy of, say, $1 \mathrm{ppm}$ is desired and an experiment requires a uniform region whose volume has a radius of $7.5 \mathrm{~cm}$ the required accuracy in the compensating coils $\left(N_{1}=49\right)$ is about 1 part in 20,000 , a rather severe requirement. On the other hand, a uniform region with $2 \mathrm{~cm}$ radius $\left(N_{1}=0.25\right)$ can be had for an accuracy of only 1 part in 1,000 in the construction of the auxiliary windings. Obviously it does not pay to compensate over a region larger than necessary.

(Paper 69C1-185) 